\title{
The status of MICE Step IV
}

\section{Yordan Karadzhov* on behalf of the MICE collaboration \\ University of Geneva \\ E-mail: yordan.karadzhovecern.ch}

Muon beams of low emittance provide the basis for the intense, well-characterized neutrino beams of a Neutrino Factory and for lepton-antilepton collisions at energies of up to several $\mathrm{TeV}$ at a Muon Collider. The international Muon Ionization Cooling Experiment (MICE) will demonstrate ionization cooling - the technique by which the mu-beam phase-space volume is reduced.. MICE is being constructed in a series of steps. At Step IV, MICE will study the properties of liquid hydrogen and lithium hydride that affect cooling. A solenoidal spectrometer will measure emittance up and downstream of the absorber vessel, where a focusing coil will focus muons. The construction of Step IV at RAL is nearing completion. The status of the project will be described together with a summary of the performance of the principal components. Plans for the commissioning and operation and the Step IV measurement programme will be described.

The European Physical Society Conference on High Energy Physics 22-29 July 2015

Vienna, Austria

${ }^{*}$ Speaker. 


\section{Ionization cooling}

Ionization cooling [1] [2] (Fig. 1) provides the only practical solution to prepare high brilliance beams necessary for a Neutrino factory [3] or Muon colliders [4], because it is fast enough to cool the beam within the muon lifetime.

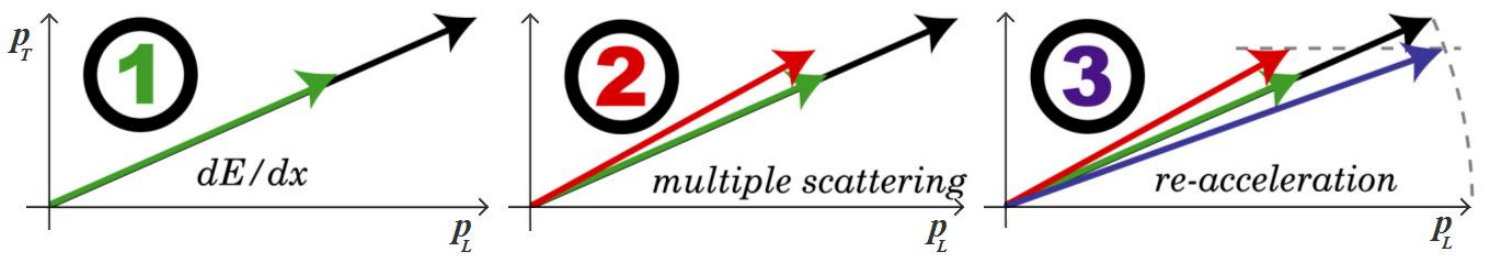

Figure 1: Ionization cooling principle: 1 . Energy loss by ionization $\left(d E / d x\right.$ reduces $P_{L}$ and $\left.P_{T}\right) 2$. Heating from multiple scattering 3. $P_{L}$ restored by RF cavities

To maximize cooling we need a material with low- $\mathrm{Z}$ to be placed at a position where the transverse momentum $P_{T}$ has a maximum ( $\beta_{\perp}$ has a minimum).

\section{MICE}

The Muon Ionization Cooling Experiment (MICE) [5] is under development at the Rutherford Appleton Laboratory (UK). The goal of the experiment is to build a section of a cooling channel that can demonstrate the principle of Ionization cooling and to verify its performance in a muon beam. The MICE collaboration consists of $\sim 150$ scientists and engineers from Bulgaria, China, Italy, Japan, Netherlands, Serbia, Switzerland, UK and USA.

MICE uses muon beams of limited intensity, where particles can be measured individually using scintillator-based detectors. The particle content of the muon beam is measured by using time-of-flight detectors (TOF0, TOF1, TOF2), Cherenkov detectors, KL (KLOE Light) calorimeter and Electron Muon Ranger (EMR), that provide precise muon, pion and electron identification. Two identical Scintillating fiber trackers in $4 \mathrm{~T}$ solenoids are situated upstream and downstream of the cooling section and will be used to reconstruct the beam emittance, by measuring the spatial coordinates and momentum $\left(x, y, p_{x}, p_{y}, p_{z}\right)$ of each muon.

The experiment is being assembled, tested and operated in steps. Each step will validate different parts of the setup. The original schedule has been re-baselined in order to allow the final demonstration of ionization cooling to be completed in 2017.

\section{Step I - completed}

The MICE muon beam line at ISIS, RAL, was built to provide beams of different momenta and emittance so that the performance of the cooling channel can be fully explored. During the initial stage of MICE, a novel technique based on time-of-flight counters was used to establish that the beam emittances are in the range $0.7-2.8 \mathrm{~mm} . \mathrm{rad}$, with central momenta from $170-$ $280 \mathrm{MeV} / \mathrm{c}$, and momentum spreads of about $20 \mathrm{MeV} / \mathrm{c}$. The beamline (Fig. 2) is now constructed and operational [7]. Its properties have been measured. The PID detectors (TOF0, TOF1, Ckov, 


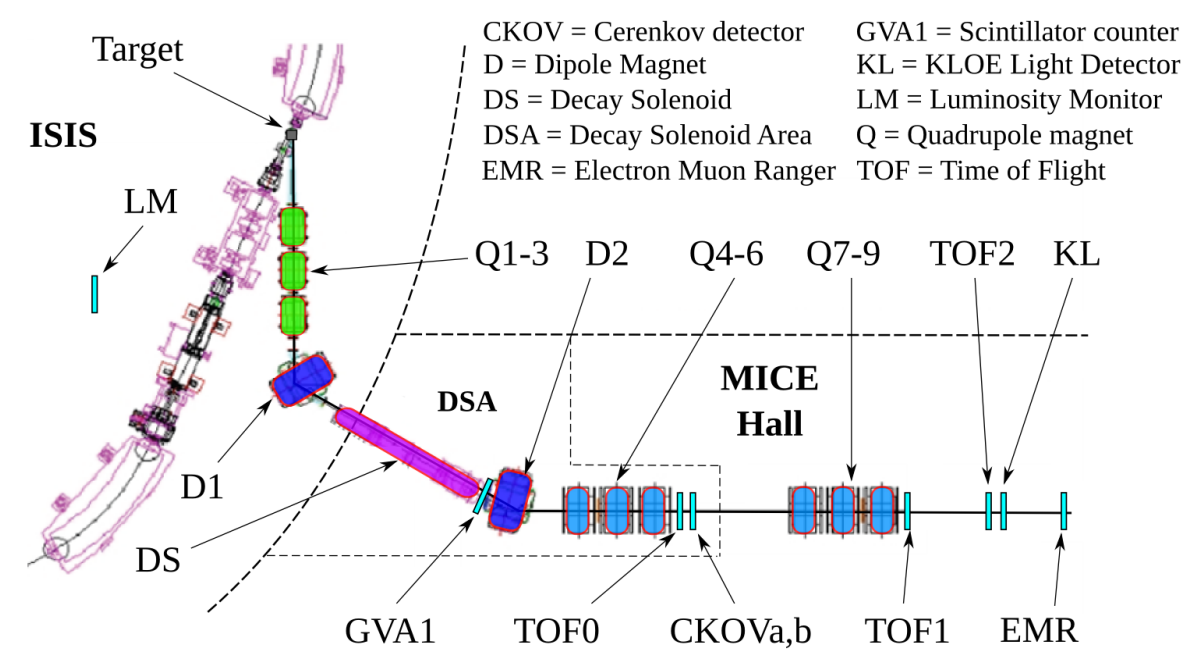

Figure 2: MICE upstream beam line.

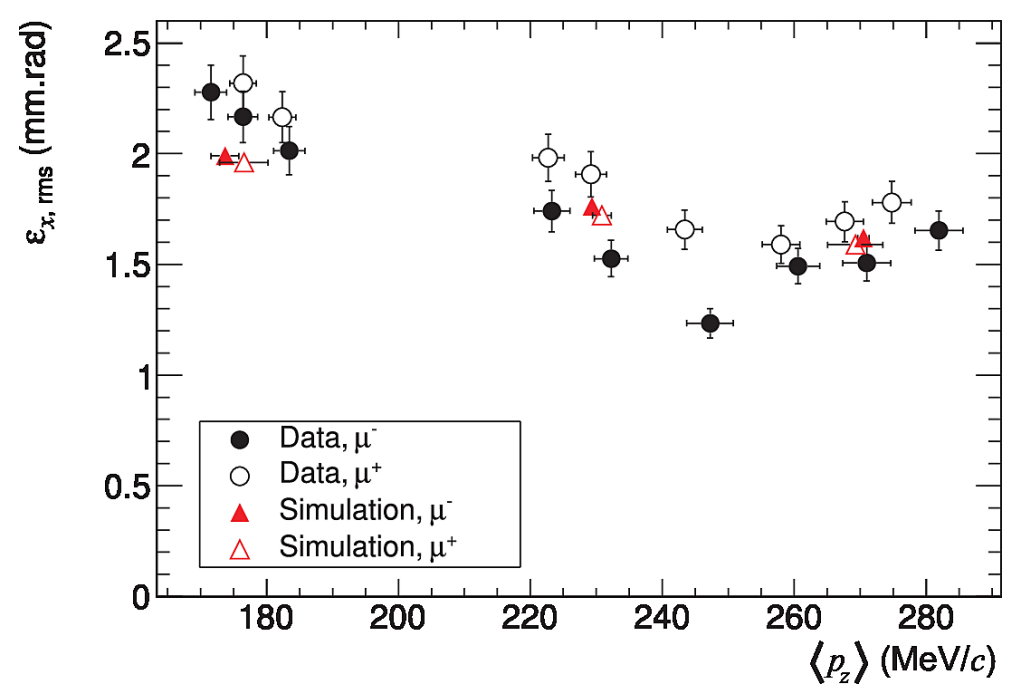

Figure 3: Horizontal emittance after correction for measurement resolution and multiple scattering versus mean $p_{z}$ of the seventeen measured beams. Solid black circles: $\mu$ data, open black circles: $\mu^{+}$data, solid red triangles: $\mu$ simulation, open red triangles: $\mu^{+}$simulation. The nominal $p_{z}=140 \mathrm{MeV} / \mathrm{c}$ beams correspond to momenta in the range $170-190 \mathrm{MeV} / \mathrm{c}, p_{z}=200$ to $220-250 \mathrm{MeV} / \mathrm{c}$, and $p_{z}=240$ to $250-290 \mathrm{MeV} / \mathrm{c}$.

TOF2, KL, EMR) are installed. They have been commissioned with $13 \times 10^{6}$ triggers collected in Step I. The MICE Muon Beam meets all the design requirements [8] (Fig. 3).

\section{Step IV - Description}

The physics plan for Step IV (Fig. 4) of the experiment includes first measurements of transverse normalized emittance reduction, measurements of the equilibrium emittance of a given absorber and for a given beta function, measurement of $6 \mathrm{D}$ emittance change, as well as precision measurements of multiple scattering. Lithium Hydride and Liquid hydrogen absorbers with differ- 
ent optics configurations will be examined. Fig. 5 shows the status of the implementation of Step IV in the MICE Hall.
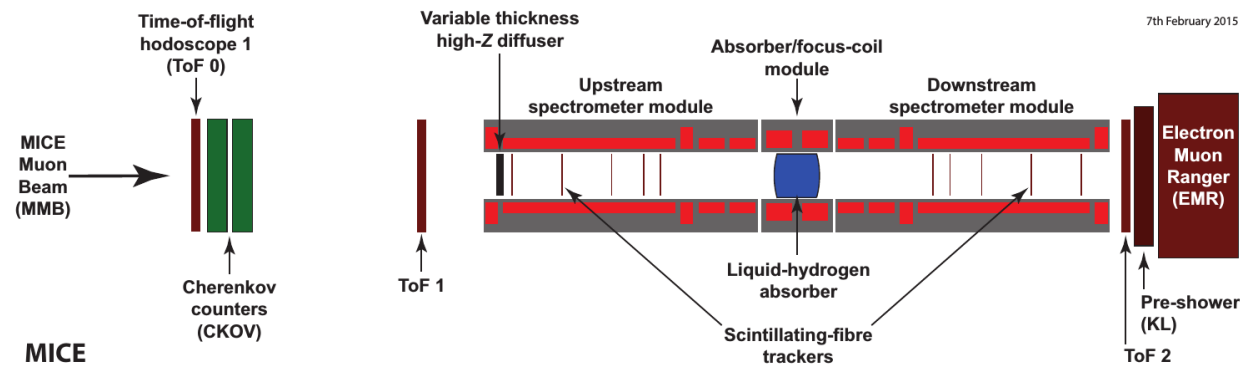

Figure 4: Schematic diagram of the Step IV configuration of MICE. The muon beam enters from the left of the figure. The beam-line instrumentation (the time-of-flight, ToF, hodoscopes, the Cherenkov (CKOV) counters, the pre-shower, KL, detector and the Electron Muon Ranger, EMR) are indicated. The spectrometer solenoids, and the scintillating-fibre trackers they contain are shown upstream and downstream of the central absorber/focus-coil (AFC) module

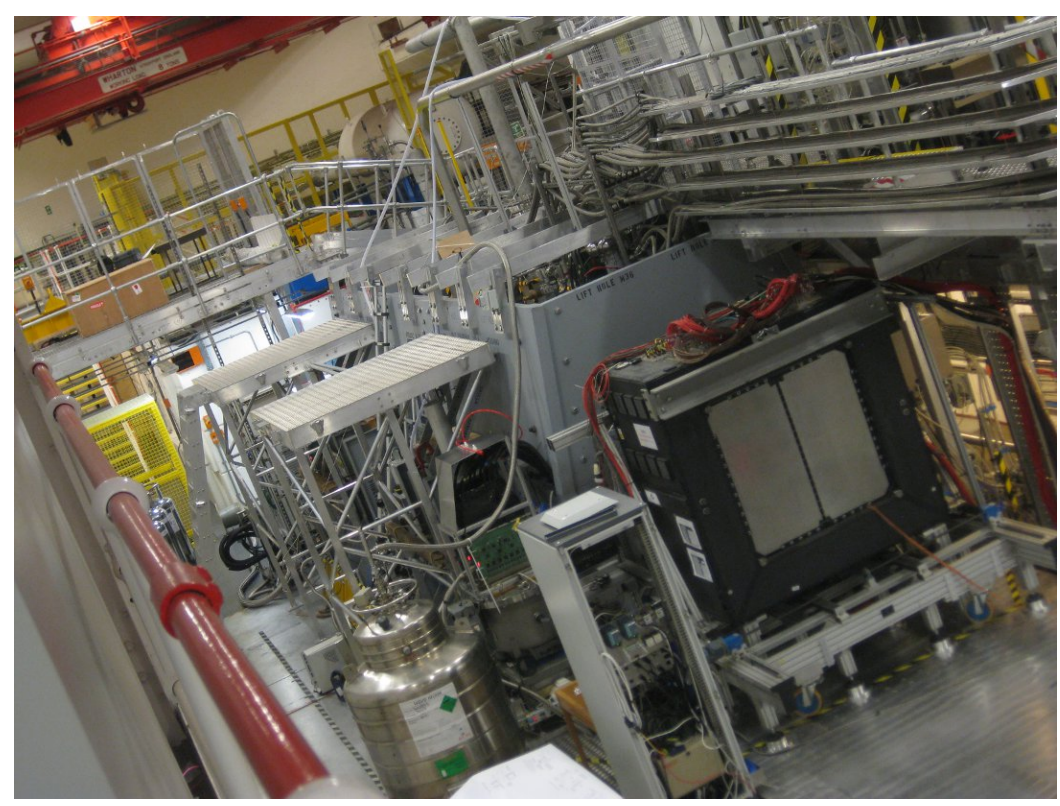

Figure 5: Photograph of the state of construction of MICE Step IV taken in August 2015. The upstream spectrometer, the absorber/focus-coil module and the downstream spectrometer, placed inside a Partial return yoke, may be seen in front of the downstream PID system (ToF2, KL and EMR).

A description of the new components introduced in Step IV is given in the following paragraphs.

\subsection{Trackers}

Each tracker module is composed of a scintillating-fibre tracking detector (Fig. 6) installed within a $4 \mathrm{~T}$ spectrometer solenoid. Each tracking detector consists of 5 planes (stations), composed 


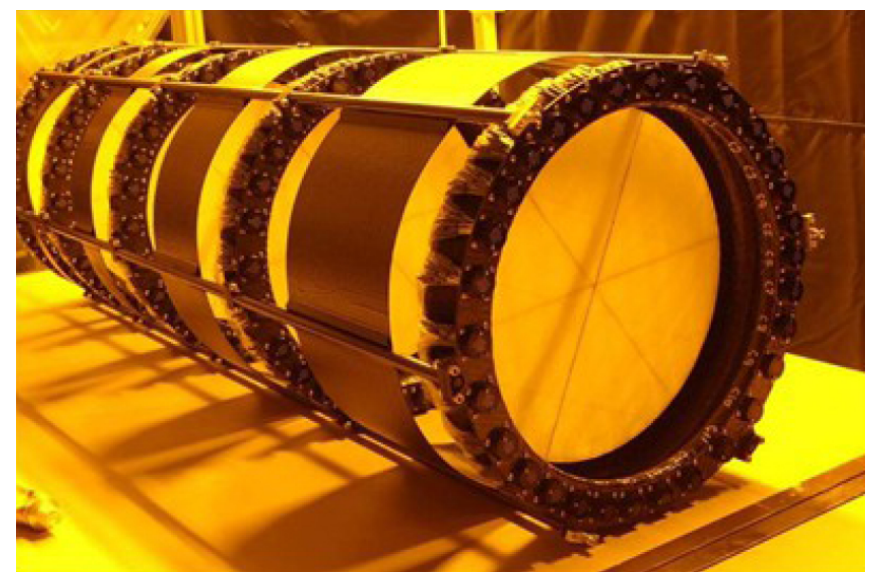

Figure 6: tracker

of three doublet-layers of scintillating fibres, providing 3 views. To reduce multiple Coulomb scattering of muons to an acceptable level, a fibre diameter of $350 \mu \mathrm{m}$ was used.

The trackers are positioned inside the bore of $\sim 1.3 \mathrm{~m}$ long superconducting solenoid, which provides a uniform $4 \mathrm{~T}$ field. Additional superconducting coils are used to match the magnetic optics to that of the the cooling channel.

\subsection{Diffuser}

The Diffuser (Fig. 7) placed before the upstream spectrometer will be used to produce a broad range of emittances. Multiple scattering in materials with small radiation lengths is used to increase the beam emittance.
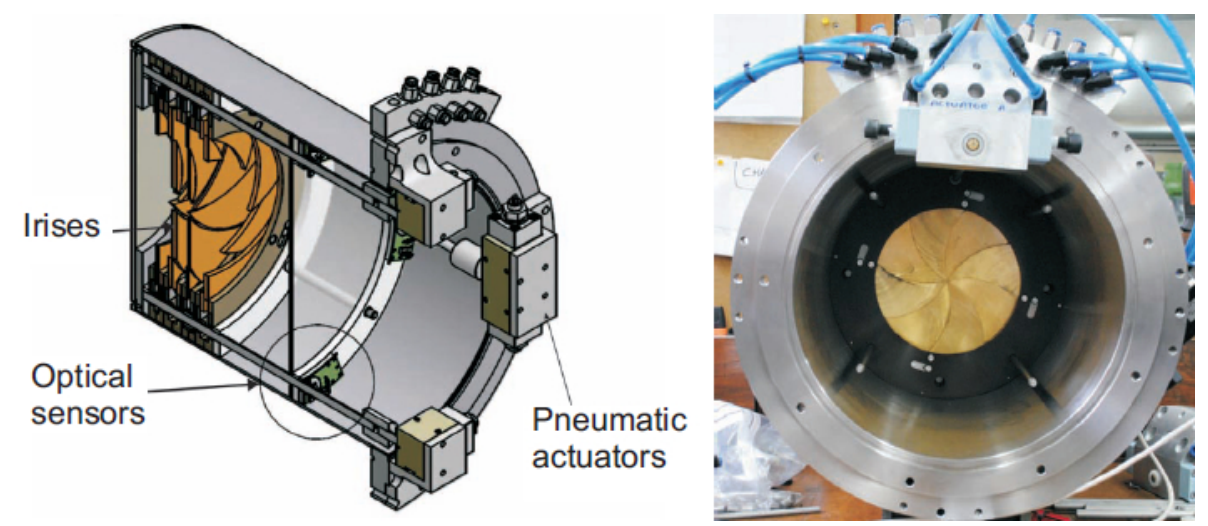

Figure 7: Left: schematic of the MICE diffuser showing the irises. Right: one of the brass diffuser irises being tested.

\subsection{Absorber/focus-coil module}

The focus-coil module (Fig. 8, right) consist of two superconducting 4T coils wound on a single bobbin. The coils can be run with the same polarity (Solenoid mode) or with opposite polarities (Flip mode) and will providing strong focusing at the absorber. The liquid-hydrogen 


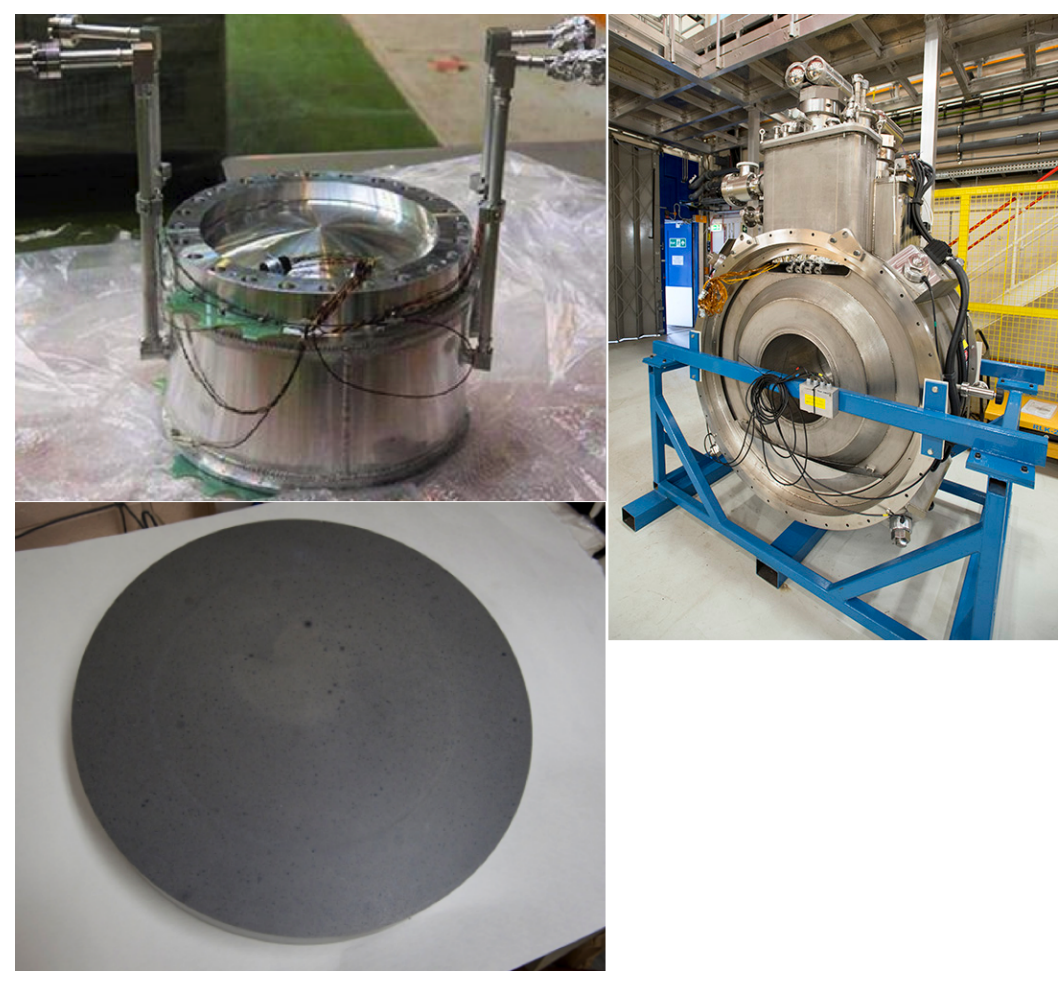

Figure 8: Left top: the liquid-hydrogen absorber vessel prior to installation in the focus-coil module. Left bottom: the lithium-hydride absorber. Right: the Focusing coil module.

absorber is shown on Fig. 2, left-top. The $65 \mathrm{~mm}$ thick disk of the lithium-hydride absorber is shown on Fig. 8, left-bottom.

\section{Conclusions}

The MICE collaboration is now on track to deliver its demonstration of ionization cooling by 2017. Step IV construction is nearly completed and data-taking will start in the autumn of 2015.

\section{References}

[1] D. Neuffer, Part. Accel. 14 (1983) 75;

[2] D. Stratakis et al.. Phys.Rev.ST Accel.Beams 16 (2013) 091001

[3] S. Choubey et al., Interim Design Report, arXiv:1112.2853

[4] C. M. Ankenbrandt et al.. Phys.Rev.ST Accel.Beams 2 (1999) 081001

[5] MICE web site http://mice.iit.edu contains detailed information about the experiment.

[6] MICE proposal, MICE note 21, (2003)

[7] MICE collab., JINST 7 (2012) P05009, arXiv:1203.4089v2

[8] D. Adams et al. [MICE Collaboration], Eur. Phys. J. C 73 (2013) 10, 2582, arXiv:1306.1509 